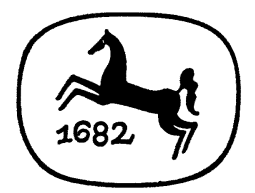

Studien zur Allgemeinen und Vergleichenden

Literaturwissenschaft 9

Herausgegeben von Eberhard Lämmert, Klaus Reichert, Karlheinz Stierle und Jurij Striedter, mitbegründet von Peter Szondi 
Hans-Jürgen Fuchs

\section{Entfremdung und Narzißmus}

Semantische Untersuchungen zur Geschichte der > Selbstbezogenheit < als Vorgeschichte von französisch » amour-propre «

J. B. Metzler Stuttgart 
Studien zur Allgemeinen und Vergleichenden Literaturwissenschaft Band 9

CIP-Kurztitelaufnahme der Deutschen Bibliothek

\section{Fuchs, Hans-Jürgen}

Entfremdung und Narzißmus: Semant. Unters. zur

Geschichte d. „Selbstbezogenheit« als Vorgeschichte von franz. amour-propre. - 1. Aufl. - Stuttgart:

Metzler, 1977.

(Studien zur allgemeinen und vergleichenden

Literaturwissenschaft; Bd. 9)

ISBN 978-3-476-00359-1

ISBN 978-3-476-03077-1 (eBook)

DOI 10.1007/978-3-476-03077-1

\section{(C) Springer-Verlag GmbH Deutschland 1977}

Ursprünglich erschienen bei J. B. Metzlersche Verlagsbuchhandlung und Carl Ernst Poeschel Verlag GmbH in Stuttgart 1977 


\section{Inhalt}

Vorbemerkung ....................... VIII

Problemstellung . . . . . . . . . . . . . . . 1

1. Darstellung und Kritik der amour-propre-Forschung . . . . . . 1

a) Bedeutungsspektrum in den Wörterbüchern des 19. und 20. Jahrhunderts . . . . . . . . . . . . . . . . . 2

b) Ertrag der wort- und geistesgeschichtlichen Forschung für die Erhellung der Bedeutungsstrukturen . . . . . . . . . 11

2. Zu Gegenstand und Methode der Untersuchung . . . . . . . . 28

I. Zur Entstehungsgeschichte der Begriffsstrukturen 'SelbstLiebe und >Eigen-Liebe im Griechischen und Lateinischen . . . . . . . . . . . . . . . . 32

1. Griechische Antike und Patristik . . . . . . . . . 32

2. >Selbst-Liebe und $>$ Eigen-Liebe im Lateinischen . . . . . . . 38

a) Antike . . . . . . . . . . . . . . . . . . . . . . . . . 38

b) Augustin und der amor privatus . . . . . . . . . . . 40 40

c) Klassisch- und spätlat. amor proprius . . . . . . . . . . . . 43

d) Mittellateinische Quellen . . . . . . . . . . . . . . 44

II. Die Wiederaufnahme im spätmittelalterlichen Italien . . 49

1. Amour de la creature und Brunetto Latinis amour de soi . . . . 49

2. Der amore proprio Jacopones da Todi und der Spiritualität des frühen Trecento ................. 52

3. Humilitas und dignitas: die propria caritate Dantes . . . . . . 63

4. Entdogmatisierung und Säkularisierung des amor proprio bei Katharina von Siena ............... 68

III. Der amor proprio als eine Stufe in der Entwicklung des neuzeitlichen Individualismus .

1. Zur Sozio- und Psychogenese des Individualismus im mittelalterlichen Italien . . . . . . . . . . . . . . . . . .

2. Der religiöse Individualismus der Mystik im Zeitalter der Bettelorden .................... 
3. Entselbstung und Introversion als zentrale Phänomene der mystischen Existenz . . . . . . . . . . . . . . . . . 82

4. Entfremdung und Narzißmus . . . . . . . . . . . 88

IV. Zunahme der Widerspiegelung konkreter Erfahrungen in der 'Selbstlieber-Idee des 15. Jahrhunderts

1. Terminologische Spaltung in volkssprachlichen und lateinischen Texten bei Jean Gerson . . . . . . . . . . . . . . . . . . .

2. Traditioneller Augustinismus und phänomenologische Selbstanalyse in der >Theologia naturalis des Ramón Sabunde . . . . . . 95

3. Nachlassen des Kampfs gegen den amor proprio in Italien . . 100

V. Umgestaltungen der Selbstlieber im Renaissance-Humanismus des 16. Jahrhunderts . . . . . . . . . . . . . . . 102

1. Apotheose des 'Eros-zu-sich-selbst di noi stessi im Florentiner Neuplatonismus . . . . . . . . 103

2. Die fehlende selbstlieber in der politischen Philosophie und in der Moralistik Italiens und Spaniens . . . . . . . . . . . 109

3. Erneuerung und Umwertung der philautia durch Erasmus . . . 112

4. Humanistische und aristokratische Formen gesteigerter Selbstbezogenheit in der philautie und dem amour de soy der französischen Renaissance . . . . . . . . . . . . . .

VI. Zunehmende Verinnerlichung des Protests in der Religiosität des 16. Jahrhunderts . . . . . . . . . . . . . . 136

1. Subtilität und spiritueller Rückzug des amor proprio bei Katharina von Genua . . . . . . . . . . . . . . . . . 136

2. Der amor propio der spanischen Mystik .. . . . . . . 142

a) Feudaler Protest in der negativ kommerzialisierten Gesellschaft 142

b) Humanismus und Mystik: die terminologische Scheidung von amor propio und amor de sí mismo . . . . . . . . . . . . 146

3. Die Integration des amour propre in das Vokabular der französischen Spiritualität . . . . . . . . . . . . . . . . .

a) Die Mortifikation des amour propre bei Briçonnet (1521/24) und seine Sublimierung im Calvinismus . . . . . . . .

b) Der Beginn der amour propre-Tradition durch Montaigne

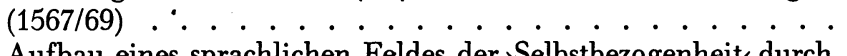

c) Aufbau eines sprachlichen Feldes der SSelbstbezogenheit durch verstärkte Ubernahme fremder Termini $(1570-1610)$. . . .

VII. 'Selbsterhaltung und 'Selbstlieber: Vives, Campanella, Hobbes . . . . . . . . . . . . . . . . .

VIII. Protest und Legitimation: Positivierungstendenzen des amour-propre als Ausdruck des erstarkenden bürgerlichen Optimismus im 17. Jahrhundert . . . . . . . . . . . 190

1. Philautie versus amour propre: sprachliche Unifizierungstendenzen im französischen Absolutismus . . . . . . . . . . . . 191 
2. Der spirituelle Raum . . . . . . . . . . . . . . . 199

a) Der shumanisme dévote und die terminologische Scheidung von amour propre und amour de soi . . . . . . . . . . . 199

b) Der Kampf gegen den amour propre als Thema des Jahrhunderts . . . . . . . . . . . . . . . . . . . . 208

c) Rearchaisierung und Dogmatisierung des Wortinhalts im Jansenismus $(1640-1660)$. . . . . . . . . . . . . .

3. Gloire und amour propre. Die Semantik der selbstlieber in

Sprache und Literatur der shonnêtes gens، . . . . . . . . . . 217

a) Religiöse Dichtung und Predigerliteratur . . . . . . . . . . 217

b) Ansätze zur Rehabilitierung der sSelbstliebe in der ersten Hälfte des 17. Jahrhunderts . . . . . . . . . . . . . . . 221

c) Von der Demaskierung des Ich zur Legitimierung des Selbsterhaltungstriebs in der Aphorismus-Literatur . . . . . . . 224

d) Das snatürlicher eigene Selbstwertgefühl in der Briefliteratur 234

e) Zunehmend aufgewertete Wortverwendungsweisen in Roman, Drama, Gedicht . . . . . . . . . . . . . . .

f) Der Durchbruch der Positivierungstendenzen oder Die Verbürgerlichung der gloire: Nicoles TTraité de la charité et de l'amour propre $(1675)$. . . . . . . . . . .

IX. Apotheose und Niedergang: Abriß der semantischen Situation von amour-propre im 18. Jahrhundert. . . . . . . 267

1. Die Aufwertung von amour-propre im Vergleich zu ähnlichen Positivierungsprozessen der deutschen und englischen Aufklärung

2. Selbstwertgefühl und Rollenzwang: der amour-propre in Selbstzeugnis, Brief, Literatur . . . . . . . . . . . . . . . 274

3. Selbsterhaltung und Gemeinwohl: die aufklärerische Apologie der >Selbstliebe und des >wohlverstandenen Interesses ‘ . . . . . 279

4. Selbstbefangenheit und Reflexion: der quietistische Kampf um die sinteressenlose Lieber . . . . . . . . . . . . . . 285

5. Selbstentfremdung als genetischer Prozeß: amour-propre und amour de soi bei Rousseau . . . . . . . . . . . . 287

Anhang: Amour-propre im 19. und 20. Jahrhundert . . . . . . . 293

Zusammenfassung und Ausblick . . . . . . . . . . . . 296

Anmerkungen ................... 312

Literaturverzeichnis . . . . . . . . . . . . . . 372

I. Wörterbücher und Nachschlagewerke . . . . . . . . . . . 372

II. Quellen . . . . . . . . . . . . . . . . . . . 375

a) Manuskripte . . . . . . . . . . . . . . 375

b) Gedruckte Texte . . . . . . . . . . . . . . 375

III. Wissenschaftliche Darstellungen . . . . . . . . . . . 382

Nachträge . . . . . . . . . . . . . . 390

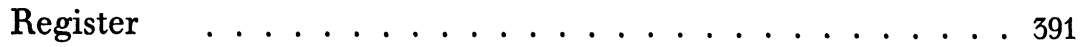




\section{Vorbemerkung}

»Le narcissisme est un processus d'aliénation bien défini: le moi est posé comme une fin absolue et le sujet se fuit en lui.«

Simone de Beauvoir,

Le deuxième sexe, II, p. 459

Zweierlei ist mit Entfremdung und Narzißmus gemeint: In der Entwicklung der sogenannten sabendländischen`Zivilisation, insbesondere während der letzten acht Jahrhunderte, wird das narzißtische Gleichgewicht des Menschen, sein Selbstwertgefühl und seine >Identitätı, durch immer stärkere Schübe gesellschaftlicher Entfremdung belastet und deformiert. Durch den so bedingten Mangel an snarzißtischer Zufuhrı, die u. a. durch soziale Geborgenheit und Verwurzelung vermittelt wird, wendet das isolierte und verunsicherte Individuum seine Libido verstärkt auf fragmentierte Teile seines Selbst. Zur Bewältigung der veränderten Lebenssituation entsteht einmal ein strengeres, Selbstzwänge verinnerlichendes Überich, zum andern ein ssekundärer Narzißmusく, der das entstandene narzißtische Defizit und die damit gegebene Bedrohung des Selbstwerterlebens dadurch abzuwehren versucht, daß psychische Gehalte früherer Entwicklungsstufen, insbesondere Omnipotenzphantasien und Symbiosewünsche, wiederbelebt werden. In diesem Sinne ist das obige Zitat Simone de Beauvoirs zu verstehen: psychologische Epiphänomene sozialer Entfremdung sind Formen eines ssekundären`, d. h. letztlich pathologischen Narzißmus. Die Zersetzung einer Gesellschaft und damit die Entfremdung und Isolierung der einst kollektiv in ihr gebundenen Individuen müßte also einhergehen mit dem epidemischen Auftreten der Symptome eines solchen >DefektNarzißmus`(G. Ammon, Dynamische Psychiatrie, 1973), die auch für historische Sprach- und Kulturwissenschaften begrenzt aufspürbar sind. Formelhaft verkürzt heißt die Leitlinie eines solchen Suchens Narzißmus als Entfremdung.

So haben etwa die Forschungen J. R. Armogathes zur ınéologie، des 18. Jahrhunderts gezeigt, wie sehr sich die zunehmende Verein- 
zelung und die soziale Verelendung des Menschen jener Epoche in der rapiden Ausdifferenzierung der Wortfelder sisolation ‘ und >égoïsme niederschlägt.

Die vorliegende Arbeit ist die gestraffte und umgearbeitete Fassung meiner unter dem Titel $\mathrm{Zu}$ Vorgeschichte und Semantik von französisch »amour-propre«. Untersuchungen zur Entwicklung einer Begriffsstruktur im sprachlichen Feld der selbstbezogenheite von den Anfängen bis zum Ende der französischen Hochklassik im SS 1973 vom Fachbereich Sprachen und Kulturen des Mittelmeerraums und Osteuropas der Justus Liebig-Universität Gießen angenommenen Dissertation. Die Anregung zu der Arbeit kam aus den kontrastiven Forschungen von Prof. Dr. H.-W. Klein zur Lexik des Deutschen und Französischen. Die Ausgangsfrage lautete: Wie kommt es, daß die offenbar nach einem sehr ähnlichen Wortbildungsschema entstandenen Komposita frz. amour-propre und dtsch. Eigenliebe in ihren gebräuchlichen Bedeutungen so stark divergieren? Die einzelnen Fragestellungen, die sich im Verlauf der Untersuchungen am Forschungsgegenstand ergaben, führten einerseits immer weiter in die antike und romanische Vorgeschichte des Wortes amour-propre. Andererseits verlangten sie immer stärker eine historisch-soziologische und anthropologische Klärung der an den sprachlichen Beständen feststellbaren Inhalte und Veränderungen. Dabei erwies sich die sSelbstlieber als ein Schlüsselbegriff bei der Konstituierung neuzeitlichen Denkens, der wie die verwandten, ebenfalls um die Vermittlung von Subjektivität und Sozialität bemühten Konzepte sSelbsterhaltung und >Interesser zwar auf Sprach- und Denkformen der griechisch-römischen Antike zurückgreift, diese aber auf spezifische, >moderne $<$ Weise weiterentwickelt.

Die Arbeit versucht also, aus der Perspektive der historischen Sprach- und Kulturwissenschaften Erkenntnisgewinne an einem Gegenstand zu machen, der innerhalb des letzten Jahrzehnts auch in den Humanwissenschaften stark in den Mittelpunkt getreten ist. Das zum "Zentralthema des allgemeinen Konfliktbewußtseins « $(\mathrm{H}$. E. Richter) gewordene sozialpsychologische Konzept der >(Ich-)Identität< (E. H. Erikson), die in der Philosophie wieder entbrannte Diskussion über 'Selbsterhaltung « und 'Subjektivität`, die in der psychoanalytischen Theoriebildung zentral gewordene >Narzißmus - -Problematik - sie alle zeigen, wie dringlich die kollektive Identitätskrise der Gegenwart ein Fragen nach dem Subjekt, seinem Selbstverständnis und den Prinzipien seiner Vergesellschaftung entstehen läßt. Die 
Frage nach dem Sprachzeichen amour-propre, nach den Bedingungen seiner Entstehung und seiner Veränderungen, ist so nur ein Sonderfall der Frage nach der 'Selbstbeziehung des Menschen unter seinen jeweiligen konkreten Lebensbedingungen.

Der psychosoziale Phänomenzusammenhang, auf den die sprachlich-literarische Fragestellung weiter verweist, wurde erstmals von Arnold Hauser (Der Manierismus, 1964) an Hand der >Krise der Renaissance $<$ mit den Konzepten `Narzißmus $<$ und `Entfremdung $<\mathrm{zu}$ erfassen versucht. Von seiten der Psychoanalyse hat vor allem Igor A. Caruso den Zusammenhang von Entfremdung und ssekundärem (pathologischem) Narzißmus unterstrichen (Soziale Aspekte der Psychoanalyse, 1972; Narzi $\beta$ mus und Sozialisation, 1976). Die Skala des epidemisch verbreiteten >defekt-narzißtischen Syndroms< reicht vom spezifisch okzidentalen $>$ Bewußtsein seiner selbst< und dessen Ideologisierung als >Individualismus über Egozentrik, Egoismus und sog. >narzißtische Persönlichkeitsstörungen ‘ bis zu Schizophrenie und Suizid. Igor A. Caruso hat eindringlich dargestellt, wie die mechanisierte, automatisierte und seelenlose Innenwelt eines rautistischen, d. h. in seinem frühen Narzißmus durch eine entfremdete Kommunikation zutiefst gestörten Kindes die äußeren Verhältnisse einer entmenschlichten und verdinglichten Welt reproduziert. Für die Sprach- und Kulturwissenschaften bieten sich zweifellos viele Wege, zur Rekonstruktion von historischen Entfremdungsprozessen, der Isolierung der Individuen sowie der daraus resultierenden Formen der kompensatorischen >Narzissierung < des Bewußtseins beizutragen. Die Wort- und Begriffsgeschichte ist nur einer davon.

Jede Frage nach einer reflexiven Beziehung des Selbst impliziert auch die Frage nach der Genese der Subjekt-Objekt-Spaltung des Bewußtseins, die die Voraussetzung dafür ist, daß das Ich sich selbst zum Objekt seines Erkennens machen kann. Nach H. Kilian (Das enteignete Bewußtsein, 1971) entstand sie als Produkt des Ubergangs von den in der Großfamilie organisierten Frühkulturen zu den klassengespaltenen Hochkulturen, die mit der Etablierung der Herrschaftsstruktur auch deren Verinnerlichung im kollektiven Bewußtsein als "herrschaftsstrukturelle Identifikation " mit sich brachten. Das Korrelat jener verinnerlichten Spaltung in soben ten auf der Ebene des Erkennens sei die Subjekt-Objekt-Spaltung. R. W. Müller hat kürzlich eine ökonomisch-epistemologische Theorie der Entstehung der Subjekt-Objekt-Spaltung vorgelegt, die auf dem behaupteten Zusammenhang von Warenform und Denkform beruht 
(Geld und Geist. Zur Entstehungsgeschichte von Identitätsbewußtsein und Rationalität seit der Antike, 1977). Die Subjekt-ObjektSpaltung ist nach Müller nur da möglich, wo in einer Gesellschaft intensiven Warenaustauschs sich immer mehr der Doppelcharakter der Ware als Gebrauchsding und Wert entfaltet. Durch das Abstraktionsprinzip des Werts, das "zu der frappanten Kristallisationsform des Münzgelds« verdinglicht, kommt es zum Gegenüber von Subjekt und Objekt der theoretischen Erkenntnis. Erst diese Subjekt-Objekt-Spaltung erzeugt die scheinbar sselbstverständlichen Kategorien der individuellen und der logischen Identität, auf denen die moderne Rationalität als abstraktes Denken und theoretisches Erkennen beruht. Die erste Frühform von Ich-Identität im Sinne einer Autonomie des Subjekts meint Müller in der Lyrik des Archilochos zu finden, der in der Mitte des 7. vorchristlichen Jahrhunderts die Folgen der neuen Form der Vergesellschaftung nach dem abstrakten Wertprinzip am eigenen Leib erfuhr. - Beide Thesen scheinen sich eher zu ergänzen als zu widersprechen. Nach einem ersten Schub an Entfremdung und damit korrelierender SubjektObjekt-Spaltung durch die Entstehung der patriarchalischen Herrschaftskulturen kommt es zu einem zweiten, sehr viel energischeren, die alte Gesellschaft zersetzenden Entfremdungsschub durch die Etablierung des Abstraktionsprinzips des Werts im Rahmen des Warenaustauschs im griechischen Raum des 7. vorchristlichen Jahrhunderts. In beiden Phasen nimmt die Fähigkeit der Naturbeherrschung mit Hilfe abstrakten Denkens und theoretischen Erkennens stark zu.

Die Geschichte der sSelbstliebe beginnt knapp zwei Jahrhunderte später, nachdem mit Archilochos eine erste Form von >modernerı, d. h. vom Kollektiv gelöster Ich-Identität aufspürbar wurde. Ich-Identität bedeutet immer auch - zumindest potentielle - IchGefährdung. E. Wulffs Untersuchungen im Bereich transkultureller Psychiatrie, die R. W. Müller ausführlich verwendet, haben gezeigt, daß der noch weitgehend im Kollektiv der Großfamilie vergesellschaftete Vietnamese in Sprache und Denken weder die individuelle Identität (>ich`, >du<) noch die logische Identität und die darauf aufbauenden Kategorien kennt. Ebenso fehlt im psychopathologischen Spektrum des Vietnamesen die für die euro-amerikanische Kultur so charakteristische Ich-Störung mit ihrer unbewältigten Ambivalenz (`Gespaltenheit`). Die ersten Indizien für diese Ich-Gefährdung in der westlichen Kultur scheinen in eben jenem 5. Jahrhundert zu 
liegen: neben den Gehalten der griechischen Tragödie ist es eine "Inflation der reflexiven Ausdrucksweise" (K. Gantar), der auch die 'Selbstliebe، ihre Entstehung verdankt, die darauf hinweist, wie das durch Zunahme sozialer Entfremdung bedrohte Ich ein immer strengeres Uberich ausbildet und sich immer stärker zum Objekt wird. So ist meistens die begriffsgeschichtlich aufspürbare sSelbstlieber zunächst Ausdruck des Protests gegen die Zersetzung der alten Gesellschaft durch eine neue Form der Vergesellschaftung, die die Beziehungen der Menschen nicht mehr nach ihren konkreten Bedürfnissen, sondern immer mehr über die Gesetze des abstrakten Tauschwerts regelt. Hauptcharakteristika bürgerlicher Gesellschaft sind Herrschaftsprinzip und das über die Abstraktion des Werts operierende Tauschprinzip, aus denen sich die weiteren (Abgrenzung, Isolation, Konkurrenz, Rationalität) ableiten.

R. W. Müller will u. a. beweisen, daß die in der griechischen Polis entwickelten Denk- und Bewußtseinsformen im wesentlichen dieselben sind, auf die sich die entwickelte bürgerliche Gesellschaft der Moderne stützen kann. In der >Ủbergangsgesellschaftく der Antike als >Keimer angelegt, konnten sie sich nach der mittelalterlichen Latenzzeit des Feudalismus durch die Wiederbelebung von Warentausch und Geldverkehr bis zur Entstehung des Handels-, Finanzund Industriekapitalismus in der Moderne voll entfalten. Seine >Kritik der Erkenntnistheorie‘ ergänzt sich so um eine >Kritik des SelbstErlebens und der Identitätsbildung ‘, in deren Mittelpunkt die historisch-genetische Erklärbarkeit der Syndrome von 'Egoismus und ,Egozentrik steht. Neuere Erweiterungen der psychoanalytischen Theoriebildung wie die >Kritische Theorie des Subjekts` (A. Lorenzer) und die >Kritik des Individualismus` (D. Spazier/J. Bopp, 1975) weisen in ähnliche Richtungen: die spezifische Erlebnisform des bürgerlichen Subjekts ist die egozentrische Identität, d. h. der sekundär-defizitäre Narzißmus. Die Diskontinuität zwischen Antike und Moderne hingegen besteht darin, daß die im Kern identischen `Keime` bürgerlicher Subjektivität durch ihre konsequente Entfaltung immer mehr zu Zerstörung und Selbstzerstörung denn zur Eröffnung positiver Potentialitäten beitragen.

Nachdem die Umarbeitung des Manuskripts im Dezember 1976 abgeschlossen war, konnten mir später bekannt werdende Publikationen aus verlagstechnischen Gründen nur noch in geringem Umfang berücksichtigt werden. Dies gilt insbesondere für Volker Gerhardts Untersuchungen zum Begriff des >Interesses`(Vernunft und 
Interesse, Diss. Münster 1976). Zur Zeit entstehen einige Arbeiten zur Geistesgeschichte des 17. Jh. in Frankreich, die u. a. auch jeweils die Problematik des amour-propre diskutieren (Jean Rohou, Littérature, idéologie et société au XVIIe s.; O. Roth, Die Gesellschaft der shonnêtes gensı bei La Rochefoucauld; D. Steland, Moralistik und Erzählkunst von Mme de Lafayette bis Marivaux).

Es ist mir an dieser Stelle nicht möglich, namentlich all denen zu danken, die mit Hinweisen, Anregungen, Unterstützung, Diskussion zur Entstehung dieses Buches beigetragen haben. Besonderen Dank schulde ich Herrn Prof. Klein (Aachen) für die Anregung und Förderung der Arbeit, sowie dem Verlag für die Geduld, mit der der Überarbeitung entgegengesehen wurde. Hinweise für diese Zweitfassung verdanke ich Herrn Prof. Mölk (Göttingen). Freundlich aufgenommen wurde ich bei Forschungsaufenthalten im Trésor de la Langue Française (Nancy), im Thesaurus Linguae Latinae (München), in der Hochschule St. Georgen (Frankfurt) und in der Maison St. Louis (S. J.) (Chantilly). Bei Problemstellungen außerhalb des Rahmens der romanischen Sprach- und Literaturwissenschaften waren mir u. a. behilflich: Prof. Peter Schunck (Mainz-Germersheim), Prof. Peter Fürstenau (Gießen), J.-R. Armogathe (Paris) sowie die Patres R. Brunet und A. Rayez (Chantilly). Der französischen Botschaft in Bonn danke ich für zwei Kurzstipendien, die mir Forschungsaufenthalte in Nancy und Paris erlaubten, der Deutschen Forschungsgemeinschaft für einen Druckkostenzuschuß. 


\title{
Abkürzungen
}

\author{
AFL Arbeitsgruppe Französische Lexikologie (Berlin) \\ ALMA Archivum latinitatis medii aevi (Bulletin Du Cange) \\ ASNS Archiv für das Studium der neueren Sprachen und Literaturen \\ Dic. Aca. Dictionnaire de l'Académie Française \\ DS Dictionnaire de spiritualité ascétique et mystique \\ DTC Dictionnaire de théologie catholique \\ FEW Wartburg, Walther von: Französisches Etymologisches Wörterbuch \\ GLLF Grand Larousse de la Langue Française \\ GRM Germanisch-Romanische Monatsschrift \\ $\mathrm{HWPh}$ Historisches Wörterbuch der Philosophie \\ IGLF Inventaire Général de la Langue Française \\ LThK Lexikon für Theologie und Kirche \\ MW Mittellateinisches Wörterbuch \\ OED Oxford English Dictionary \\ PG Patrologiae cursus completus. Series Graeca. \\ PL Patrologiae cursus completus. Series Latina. \\ PMLA Publications of the Modern Language Association of America \\ RAM Revue d'Ascétique et de Mystique \\ RF Romanische Forschungen \\ RHLF Revue d'Histoire Littéraire de la France \\ SVEC Studies on Voltaire and the eighteenth Century \\ TLF Trésor de la Langue Française \\ TLG Thesaurus Linguae Graecae \\ TLL Thesaurus Linguae Latinae \\ ZfSL Zeitschrift für französische Sprache und Literatur \\ $\mathrm{ZRPh} \quad$ Zeitschrift für romanische Philologie
}




\section{Technische Vorbemerkung}

Im Text der Arbeit wurde versucht, metasprachliche Verhältnisse so konsequent wie möglich durch folgende Differenzierung anzuzeigen:

- Kennzeichnung des Worts, bzw. des Wortkörpers durch Kursivdruck;

- Kennzeichnung der Bedeutung (Wortinhalt) durch » «;

- Kennzeichnung des Begriffs durch « «.

In den Überschriften und kursiv gesetzten Passagen wird das Wort durch " " gekennzeichnet.

Kursiv gesetzte Wörter in Zitaten sind - wenn nicht anders vermerkt - Hervorhebungen des Verfassers.

Die in [ ] erscheinenden Zahlen verweisen auf die Anmerkungen. 